\title{
Effect of oral sugar solution for reducing pain in infants underwent diphtheria, pertussis, tetanus (DPT) im- munization: a randomized, double-blind controlled trial
}

\author{
Arief Priambodo, Madarina Julia, Djauhar Ismail
}

\begin{abstract}
Background Infants are often subjected to painful procedures such as diphtheria, pertussis, tetanus (DTP) immunization. Despite its negative effects, pain in infants has not got enough attention. Sweet oral solution has analgesic effect.

Objective To determine whether oral sugar solution can reduce the duration of crying in infants who got DTP immunization.

Methods This was a randomized, double-blind controlled trial performed at Growth and Development Clinic of Dr. Sardjito General Hospital and two Primary Health Centers in Yogyakarta. Study subjects were 4-6 month-old infants who got the $3^{\text {rd }}$ DTP immunization. Subjects were randomly allocated to receive $2 \mathrm{ml}$ $75 \%$ oral sugar solution (intervention group) or $2 \mathrm{ml}$ drinking water (placebo) just before the immunization. Crying was recorded from just before the injection until 3 minutes after.

Results Eighty-six subjects were enrolled; 42 subjects received sugar solution and 44 subjects received placebo. Sugar solution reduced the median duration of first cry about 38 seconds or $32 \%$ $(\mathrm{P}=0.03)$ and reduced the median duration of total crying about 35 seconds or $24 \%(\mathrm{P}=0.02)$.

Conclusion Administration of $2 \mathrm{ml} 75 \%$ oral sugar solution can alleviate pain associated with DTP immunization as shown by reduced duration of crying. [Paediatr Indones 2008;48:23-27].
\end{abstract}

Keywords: infant analgesia, immunization, pain, sugar

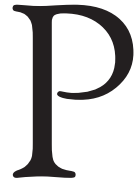
ainful DTP (diphtheria, tetanus, pertusis) immunization is one of the many routine medical procedures given to infants. Like any other pain caused by medical procedures, the pain elicited by this procedure has never really been given much attention. ${ }^{1}$ Pain associated with medical procedures in infants had got less attention because of the assumption that infants do not feel as much pain as toddlers or older children do. Or, even if they do, the pain does not yield any negative effects. 2,3

Effort to overcome or to lessen pain felt by an infant is actually very important for ethical reasons and minimizing negative effects of the pain itself. Pain in infants may lead to hypoxemia, haemodynamic instability, or increased intracranial pressure. ${ }^{4}$ A study discovered that pain associated with neonatal circumcision without local anesthetic-affected the babies' later behavior and wake-sleep cycle. ${ }^{5}$ Taddio et

From The Department of Child Health, Medical School, Gadjah Mada University, Yogyakarta, Indonesia.

Reprint requests to: Madarina Julia, MD, Department Of Child Health, Gadjah Mada University, Sardjito Hospital, Jl. Kesehatan, Sekip, Yogyakarta, 55281, Indonesia. Tel. 62-274-489726/62-274-561616. Fax. 62-274-583745. Email: madarina@pediatric-gmu.org,madarinajulia@yahoo.com 
$a l^{6}$ also noted that procedure of neonatal circumcision without analgestic had long term effects on the response toward pain inflicted by intramuscular immunizations given at 4 to 6 months later, as shown by the longer duration of crying.

These evidences have lead to the application of various methods to lessen pain in infants going through medical procedures. Several methods have been tried, i.e. the application of topical analgesic cream on the skin before procedure, giving pacifiers or sweet oral solutions. ${ }^{4,7,8}$ Of the various ways available, giving sweet solution may be the best alternative because it is easy, safe, and has swift effects. It is, therefore, ideal for minor procedures such as blood sampling or intramuscular injection. ${ }^{1}$ Sucrose solution has analgesic effect because its sweet flavor stimulates the excretion of opioid from the opioid cerebral endogen. ${ }^{9}$ Studies on analgestics for infants normally use the duration of crying as a measurement of analgesic effect. Even though there are other factors that can cause infants to cry, many investigators still use the presence or the duration of crying to measure or to evaluate pain. ${ }^{10}$ The purpose of this study was to find whether giving oral sugar solution can reduce the duration of crying in infants who got DTP immunization.

\section{Methods}

Subjects of this study were 4 to 6 month-old-infants who got whole-cell DPT vaccine. DTP immunization was performed at the Growth and Development outpatient clinic of Dr. Sardjito General Hospital and two Public Health Centers in Sleman, i.e. Puskesmas Depok I and Depok II. This study was conducted from March until December 2004 and had been approved by the Ethical Committee of Faculty of Medicine, Gadjah Mada University.

Infants were included if they were: (1) to be given the third DTP immunization and (2) 4 to 6 months old. Only infants who got to the third DTP immunization were included because response to pain were known to be associated with previous exposure to the same or similar pain. ${ }^{6}$ Infants were excluded if they: (1) born prematurely, (2) born with low birth weight ( $<2500$ gram), (3) had previous history of hospitalization, (4) were given other intramuscular immunization, e.g. HiB immunization, (5) were given paracetamol, or (6) were suspected to suffer from Down Syndrome or other congenital defects. Infants were also excluded if they had stomatitis, using pacifier or breast fed during the immunization procedure. Informed consents were obtained from the parents.

This study compared sugar solution to placebo. Subjects were randomly allocated into sugar or placebo group using a random table in block of six. Every location of the study had its own random lists. Calculation of sample size was performed using estimation of clinically significant difference of 13 seconds and a standard deviation of 21 seconds. ${ }^{1}$ With significance level of $95 \%$ and power of $80 \%$, a minimum of 41 subjects were needed for each group.

The output measured in this study was the duration of crying, since it was the easiest and the most commonly used proxy for measuring infants pain. $1,8,10-13$ The duration of crying was divided into two parameters: (1) The duration of the first cry (in seconds), which was the duration of crying from the start of the procedure until the first - lasted more than 5 seconds - stops; and (2) The total duration of crying (in seconds), which was the total duration of crying heard within 3 minutes from the start of the procedure.

Eligible infants were randomly allocated to receive either sugar solution or placebo. Sugar solution was prepared by mixing 75 gram sugar in $100 \mathrm{ml}$ drinking water. The $75 \%$ sucrose solution was used because this was the highest sugar concentration reported which could be safely given to infants. ${ }^{8}$ Higher concentration facilitated detection of effect when it existed. The placebo solution consisted of plain water. The preparation of sugar solution was done everyday.

About 2 minutes prior to DTP injection, a nurse dropped $2 \mathrm{ml}$ of the solution using a syringe into the infant's mouth, then, gave the DTP immunization intramuscularly on the infants' anterolateral thigh. Since DTP immunization was usually given along with oral polio vaccine, the polio vaccine was given after the documentation of crying was over. Sound recording via a tape recorder began from a few seconds before injection until 3 minutes after injection.

Data were entered and analyzed using SPSS for Windows (Version 10, SPSS, Chicago, Illinois, USA). The difference in the duration of crying between the intervention group and placebo was statistically analyzed. The duration of the first cry was compared 
using survival analysis and Log Rank test to accommodate the possibility of the existence of subjects who had not finished crying after the process of sound recording was stopped. For the total duration of crying, student- $t$ test or Mann-Whitney-U test was used to statistically compare the results of the two groups. The decision to use either parametric or nonparametric test was related to the distribution of the data obtained. The normality of the distribution of the data was tested using the Kolmogorov-Smirnov test. Statistical significance was set to confidence limit of $95 \%(\mathrm{P}<0.05)$.

\section{Results}

This study enrolled 86 subjects: 42 subjects of the intervention group and 44 of in the placebo group. The baseline characteristics of the subjects and their parents are listed in Table 1 . No significance differences in the baseline characteristics were observed between infants in the intervention and placebo group.

Comparison of the duration of first cry between the two groups was presented in a Kaplan-Meier survival curve (Figure 1). Overall, it could be seen that the curve for the duration of first cry in the study group was under the curve of the placebo group. The

Tabel 1. The baseline characteristics of the study subjects and their parents

\begin{tabular}{lcc}
\hline Characteristics & $\begin{array}{c}\text { Sugar } \\
\text { solution } \\
(\mathrm{n}=44)\end{array}$ & Placebo \\
& & \\
\hline Sex & & \\
$\quad$ Boys (\%) & $29(69)$ & $23(52)$ \\
Age (\%) & $15(36)$ & $25(57)$ \\
4 months & $18(43)$ & $11(25)$ \\
5 months & $9(21)$ & $8(18)$ \\
6 months & $3250(377)$ & $3066(416)$ \\
Mean (SD) birth weight (g) & $7095(934)$ & $7017(797)$ \\
Mean (SD) current weight (g) & & \\
Birth order (\%) & $14(33)$ & $19(43)$ \\
$\quad$ st child & $28(67)$ & $25(57)$ \\
$\quad$ Not 1st child & $29(5)$ & $27(5)$ \\
Mean (SD) mother's age (year) & $30(7)$ \\
Mean (SD) father's age (year) & $32(7)$ & \\
Mother's education (\%) & & $33(75)$ \\
$\quad$ High school or less & $33(79)$ & $11(25)$ \\
$\quad$ University education & $9(21)$ & \\
Father's education (\%) & & $39(68)$ \\
$\quad$ High school or less & $28(67)$ & $14(32)$ \\
$\quad$ University education & $14(33)$ & \\
\hline
\end{tabular}

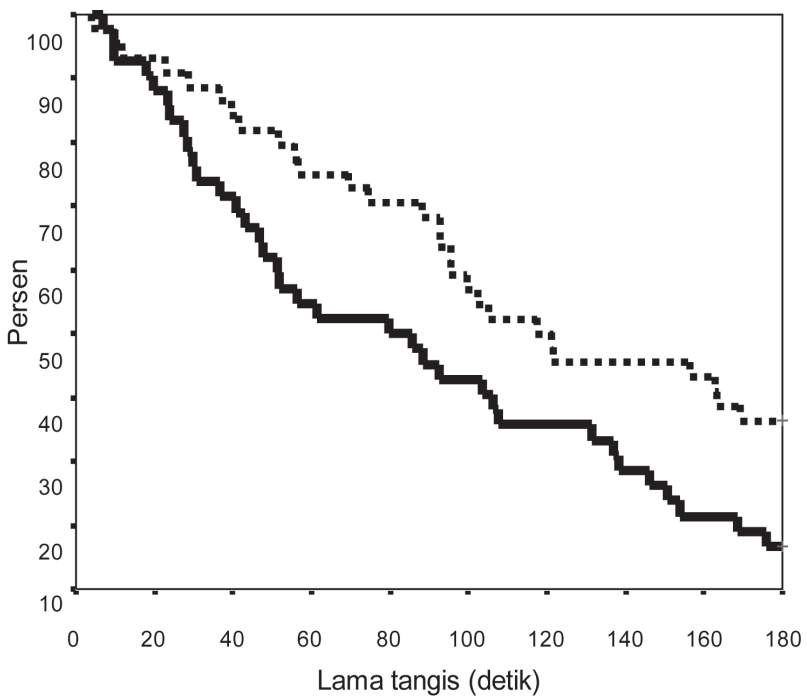

Figure 1. Kaplan-Meier survival curve on the duration of first cry, between the intervention and the placebo group.

first cry of the intervention group stopped sooner than that of the placebo group. Infants of the sugar solution group had a shorter median duration of cry compared to those given placebo, the decrease in the duration of first cry, using survival analysis, was 38 seconds or about 32\% $(\mathrm{P}=0.03)$. The number of censored observations, i.e. the number of infants who were still crying after three minutes, was larger in the placebo group; 16 (36\%) infants, versus 7 (17\%) infants in the study group.

The intervention group had a median total duration of cry of 108 seconds. This was $24 \%$ shorter than that of the placebo group, which was 143 $(P=0.02)$. Since the total duration of cry in the two groups was not normally distributed, we used a nonparametric test (Mann-Whitney-U test) to assess the difference between the two groups.

\section{Discussion}

Lewindon et al ${ }^{1}$ observed $31 \%$ decrease in the duration of first cry after administration of $75 \%$ sucrose solution. The decrease in the duration of first cry in our study, was $32 \%$, was comparable to that of Lewindon's. ${ }^{1}$ The result of this study also confirmed the results reported by Haouari et $a l^{8}$ in 1995. However, Haouari's study ${ }^{8}$ did not state the percentage of the decrease. 
Blass et $\mathrm{al}^{12}$ in a study on analgesic effect of $12 \%$ sucrose solution in 1 to 3 days old infants undergoing blood sampling procedures found a shorter survival curve in infants receiving $12 \%$ sucrose solution, showing that infants receiving sucrose solution cried for shorter duration than those receiving placebo. However, this study did not state whether the difference in the duration of first cry reached statistical significance.

There were several subjects who had not stopped their first cry when the three-minutes recording were stopped. In these subjects, the duration of cry was considered to be 180 seconds, equal to the duration of the recording process. Hence, the data on the duration of first cry in these subjects were not the actual data or numbers (censored observation). This study observed that the proportion of subjects still cried when the recording stopped was larger in the placebo group (36\%), compared to the intervention group (17\%).

Lewindon et $a l^{1}$ and Hauoari et $a l^{8}$ had also measured the total duration of cry heard in the first three minutes. Lewindon's study ${ }^{1}$ observed 38\% reduction in the total duration of cry in infants receiving $75 \%$ sucrose solution. Hauoari's study ${ }^{8}$ also showed a decrease in the total duration of cry in infants who received $12.5 \%, 25 \%$, and $50 \%$ sucrose solutions, but it did not state the amount of percentage of the decrease.

Compared to our study, Lewindon et al ${ }^{1}$ observed larger effect in sucrose solution group (on reduction in the total duration of crying). However, our study observed comparable result to that of Lewindon's ${ }^{1}$ (on the duration of first cry). This result explained that the cry related to pain felt by an infant was actually the first cry, while the other cry was more related to other conditions beside pain, such as feeling uncomfortable, fear, heat or cold. ${ }^{10,13}$ The atmosphere during immunization might induce factors affecting the total duration of crying, infants might cry due to fear, heat, or other discomforts, and not because of the pain itself. The atmosphere during immunization in this study might be different from that of Lewindon's ${ }^{1}$, leading to the difference in the decrease in percentage of the total duration of crying between the two studies.

Compared to other methods for reducing pain, i.e. topical analgestic or pacifier, giving sweet solutions such as sugar solution can be considered as an alternative because it is easy to use, safe, and has swift effect. It is therefore ideal for minor procedures such as blood sampling or intramuscular injections. ${ }^{1}$

Taddio et al ${ }^{7}$ in 1994 observed that giving topical lidocaine and prilocaine in infants receiving DTP immunization reduced the median duration of first cry by as much as $21 \%$ and reduced the median duration of total cry by as much as $59 \%$. For the duration of first cry, the median reduction of topical analgestic was inferior to that of sucrose solution in Lewindon's study ${ }^{1},(21 \%$ vs. $31 \%)$ or even to sugar solution in our study, (21\% vs. $32 \%) .{ }^{6}$ However, for the total duration of crying, the median reduction of topical analgestic was higher than that of sucrose or sugar solutions, $59 \%$ in topical analgestic, compared to $38 \%$ in sucrose and $24 \%$ in sugar. Again, the difference in the median reduction of first cry and the total duration of crying might be related to other conditions beside pain, which was not improved by the administration of sugar solution.

Referring to the duration of first cry, this study showed that the analgesic effect of topical analgestics was inferior to that of sweet solutions. This result was supported by Gradin et $\mathrm{al}^{14}$ in a study on newborns receiving vein punctures. Gradin ${ }^{14}$ reported 30\% greater decrease in the duration of crying in subjects receiving glucose solution compared to those receiving topical lidocaine and prilocaine. Topical lidocaine and prilocaine have unwanted side effect such as, erythematous skin reaction which, in Taddio's study ${ }^{6}$, occurred in $90 \%$ of the subjects. The use of topical analgestic was also considered impractical since the application must be performed at least 60 minutes before the procedure. ${ }^{7}$

While using pacifiers might have a good analgesic effect, it has many drawbacks. The use of pacifiers in infants is not in accordance with WHO program on support of exclusive breastfeeding. In the "ten steps for successful breastfeeding," particularly step number 9 , it is forbidden to give artificial nipples or pacifiers to breastfed infants because pacifier is associated with early weaning from breastfeeding. ${ }^{15-17}$

In conclusion, our study observed that administration of oral sugar solution leads to $32 \%$ reduction in the duration of cry in infants receiving intramuscular DTP immunization. Therefore, we recommend administration of $2 \mathrm{~mL}$ of $75 \%$ oral sugar solution given just prior a painful procedure. 
Arief Priambodo et al: Oral sugar solution for reducing pain in DPT immunization

\section{References}

1. Lewindon PJ, Harkness L, Lewindon N. Randomized controlled trial of sucrose by mouth for the relief of infant crying after immunization. Arch Dis Child 1998;78:453-6.

2. American Pain Society, American Academy of Pediatrics. The assessment and management of acute pain in infants, children, and adolescents. Pediatrics 2001;108:793-7.

3. Robieux IC, Kumar R, Radhakrishnan S, Koren G. Assessing pain and analgesia with a lidocaine-prilocaine emulsion in infants and toddler during venipuncture. J Pediatr 1991; 118:971-3.

4. Carbajal R, Chauvet S, Couderc S, Oliver-Martin M. Randomised trial of analgesic effects of sucrose, glucose, and pacifiers in term neonates. BMJ 1999;319:1393-9.

5. Anand KJS, Hickey PR. Pain and its effect in the human neonate and fetus. N Eng J Med 1987;317:1321-9.

6. Taddio A, Goldbach M, Ipp M, Stevens B, Koren G. Effect of neonatal circumcision on pain responses during vaccination in boys. Lancet 1995;345:291-2.

7. Taddio A, Nulman I, Goldbach M, Ipp M, Koren, G. Use of lidocaine-prilocaine cream for vaccinatiion pain in infants. J Pediatr 1994;124:643-8.

8. Haouari N, Wood C, Griffiths G, Levene M. The analgesic effect of sucrose in full term infants: a randomized controlled trial. BMJ 1995;310:1498-500.
9. Ren K, Blass EM, Zhou QQ, Dubner R. Suckling and sucrose ingestion suppress persistent hyperalgesia and spinal Fos expression after forepaw inflammation in infant rats. Proct. Natl. Acad. Sci. USA 1997;94:14715.

10. McGrath PJ, Unruh AM. Pain in children and adolescents. Amsterdam: Elseviers Science Publishers; 1987.

12. Blass EM, Hoffmeyer LB. Sucrose as an analgesic for newborn infants. Pediatrcics 1991;87:215-8.

13. Runefors P, Arnbjornsson E, Elander G, Mechelsson K. Newborn infants' cry after heel-pick: analysis with sound spectogram. Acta Paediatr 2000;89:68-72.

14. Gradin M, Erikson M, Holmqvis G, Holstein A, Scholin J. Pain reduction at venipuncture in newborns: oral glucose compared with local anesthetic cream. Pediatrics 2002; 110:1053-7.

15. Campbell C. Letters: Analgesic effects of sweet solution and pacifiers in term neonates. BMJ 2000;320:1002-4.

16. Wong SW. Electronic responses to: randomized trial of analgesic effects of sucrose, glucose, and pacifiers in term neonates. BMJ 1999. Electronic Letter Published.

17. Victoria CG, Behaque DP, Barros FC, Olinto MTA, Weiderpass E. Pacifier use and short breastfeeding duration: Cause, consequnce, or coincidence? Pediatrics 1997;99:44553. 